\title{
Atypical Intratemporal Meningioma Masquerading as Otitis Media-Clinical and Radiological Diagnostic Dilemma
}

\author{
Mrinal Matish ${ }^{1} \quad$ Abhishek Rathi ${ }^{1} \quad$ Manasi Moon ${ }^{1}$ \\ ${ }^{1}$ Department of Radiologist, Galaxy Vidarbha Diagnostic Centre, \\ Nagpur, Maharashtra, India
}

Address for correspondence Dr. Mrinal Matish, Flat No. 703, Jai Kalpana Apartment, Ramdaspeth, Nagpur 440 010, Maharashtra, India (e-mail:drmrinalmatish@gmail.com).

\begin{abstract}
Meningiomas are the most common intracranial extra-axial neoplasms with mostly straightforward radiological diagnosis; however, they can have unusual clinical and imaging manifestations posing diagnostic dilemma for radiologists and clinicians. The objective of this case report is to highlight the infrequent and misleading presentations of meningiomas to make an accurate diagnosis. A 65-year-old male patient presented

Keywords

- facial nerve

- high-resolution computed tomography

- magnetic resonance imaging

- meningioma

- otitis media with complaints of left recurrent otitis media and facial nerve palsy for past 6 months. On imaging, an aggressive predominantly cystic intracranial neoplasm was found in the left temporal fossa extending into the middle and external ear. Histopathological examination of the excised tumor revealed WHO grade I meningothelial meningioma. Meningiomas can show atypical imaging features such as large meningeal cysts, heterogenous or ring enhancement and aggressive features such as bone erosion. They can invade the middle ear and should be thought of while dealing with nonresponsive cases of otitis media.
\end{abstract}

\section{Introduction}

Meningiomas are the most common dural-based neoplasms of the central nervous system (CNS) with a relatively easy diagnosis on imaging. ${ }^{1}$ They are usually asymptomatic and detected as an incidental radiological finding. When large in size, they can present with nonspecific symptoms such as headache, paresis, and changes in mental status. Their most common location is supratentorial involving the parasagittal region, convexities, and less commonly the sphenoid ridge. Majority of meningiomas have typical imaging features on axial imaging and few have well-described atypical imaging features. Few rare meningiomas can be aggressive and present as cystic masses. The clinicians and the radiologists need to be aware of such variant meningiomas to avoid delay in diagnosis or misdiagnosis. We report such a novel case of

published online August 4, 2021
DOI https://doi.org/

$10.1055 / \mathrm{s}-0041-1734410$ ISSN 0971-3026 atypical aggressive cystic meningioma extending into the mastoid, middle and external ear from the middle cranial fossa through the tegmen tympani causing recurrent otitis media and facial nerve palsy.

\section{Case History}

A 65-year-old male patient presented with complaints of recurrent discharge from left ear and gradually progressive left-ear deafness for past 6 months. The patient also developed deviation of mouth to the right for past 1 month. Otoscopy revealed fullness in the external auditory canal and middle ear with nonvisualization of tympanic membrane. Physical evaluation including the fiberoptic examination of the nasopharynx was otherwise normal. Audiometric studies showed a conductive hearing loss with an air-bone gap

\footnotetext{
(C) 2021. Indian Radiological Association.

This is an open access article published by Thieme under the terms of the Creative Commons Attribution-NonDerivative-NonCommercial-License, permitting copying and reproduction so long as the original work is given appropriate credit. Contents may not be used for commercial purposes, or adapted, remixed, transformed or built upon. (https://creativecommons.org/licenses/by-nc-nd/4.0/).

Thieme Medical and Scientific Publishers Private Ltd. A-12, Second Floor, Sector -2, NOIDA -201301, India
} 
of $40 \mathrm{~dB}$ HL. A high-resolution computed tomography of petrous and temporal bones was performed to assess the status of middle ear and mastoid.

The CT study revealed an ill-defined soft tissue density space occupying lesion (SOL) in the left middle cranial fossa eroding the tegmen tympanum and tegmen mastoidium extending into the external and middle ear. Ear ossicles were intact. Sclerosis and hyperostosis of left squamous and mastoid temporal bone were also noted with complete soft tissue opacification of the left mastoid air cells (-Fig. 1). There was also dehiscence of tympanic and mastoid segments of facial nerve canal with extension of soft tissue within (-Fig. 2).

Magnetic resonance imaging (MRI) brain with contrast was advised for further evaluation of the left temporal fossa SOL. It revealed a large mixed solid-cystic lesion in the left temporal fossa with significant perilesional white matter edema and mass effect ( - Fig. 3). On imaging, it could not be ascertained with confidence whether the lesion was entirely extra-axial. The solid component was isointense on T1 and T2-wighted images and showed homogeneous contrast enhancement. The cystic component showed thick peripheral enhancement with multiple enhancing septae within ( - Fig. 4A and B ). There was no diffusion restriction or bleed within the lesion. Inferiorly, the lesion was extending into the left mastoid air cells, middle ear, and inner two-thirds of external auditory canal.

Based on the above imaging findings, diagnosis of an aggressive intracranial neoplasm with intra-auricular extension was made. Differentials of a high-grade glioma and metastasis from an unknown primary were considered. Paragangliomas such as glomus jugulare and jugulo-tympanicum usually present as aggressive bone eroding enhancing tumors but the location of the tumor, absence of serpiginous vessels which give rise to the typical "salt and pepper appearance" and cystic changes suggested otherwise. The patient subsequently underwent left

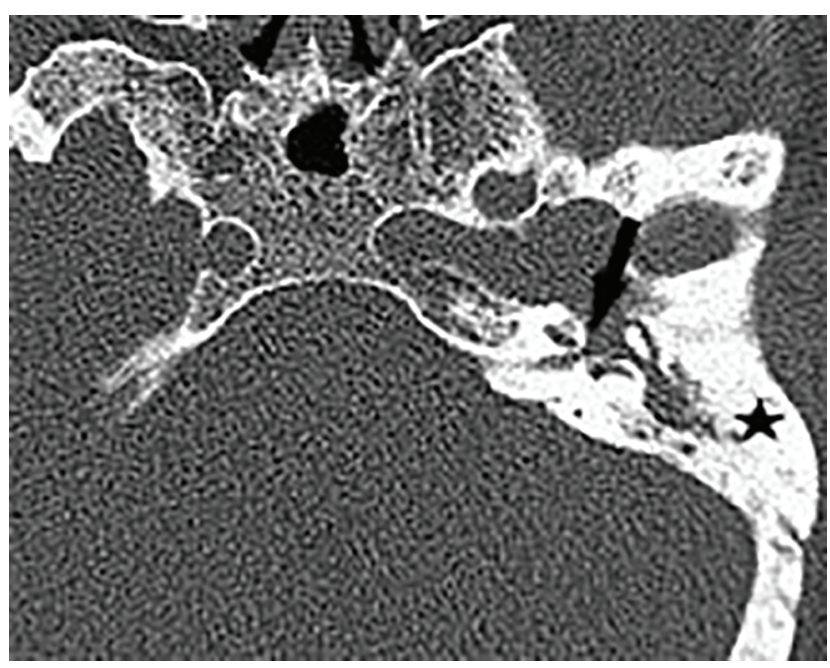

Fig. 1 Axial CT image of left temporal bone in bone window showing soft tissue density in the middle ear causing dehiscence of facial nerve canal (arrow). Hyperostosis of mastoid and squamous temporal bone also noted (asterix). CT, computed tomography. fronto-temporo-parietal decompressive craniectomy and near complete excision of the SOL. The postoperative course was uneventful. Histopathological examination of the excised tumor revealed WHO grade I meningothelial meningioma (-Fig. 5). Patient was advised clinico-radiological follow-up.
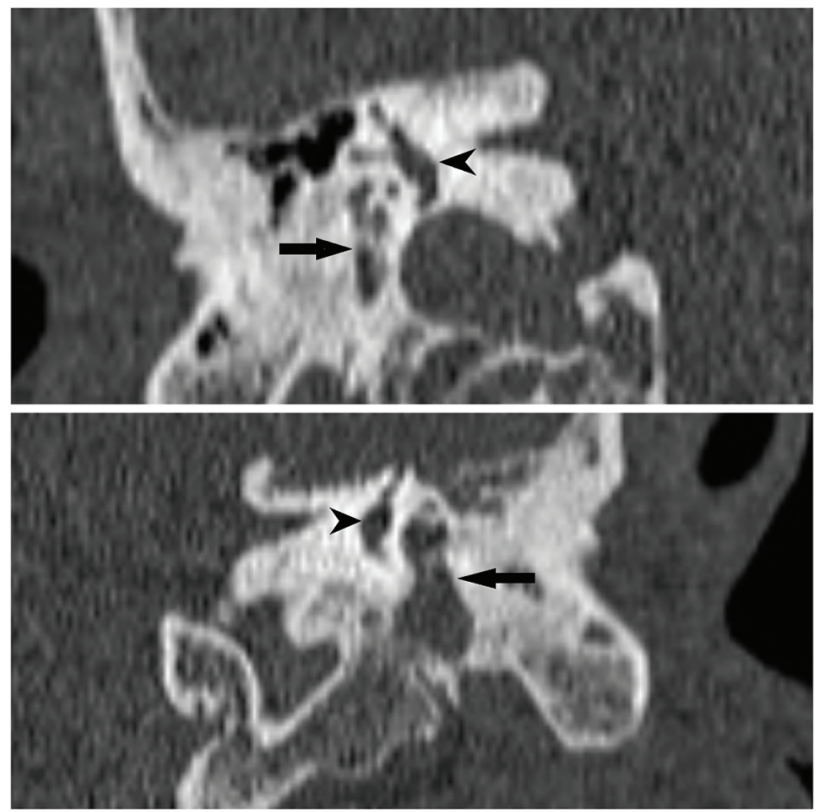

Fig. 2 Coronal CT image (magnified view) in bone window setting at the level of vestibule (arrowheads). Panel (A) shows normal caliber of mastoid segment of facial nerve canal on the normal right side (arrow). Panel (B) shows the mastoid segment of facial canal on the left is dilated and filled with soft tissue (arrow). CT, computed tomography.

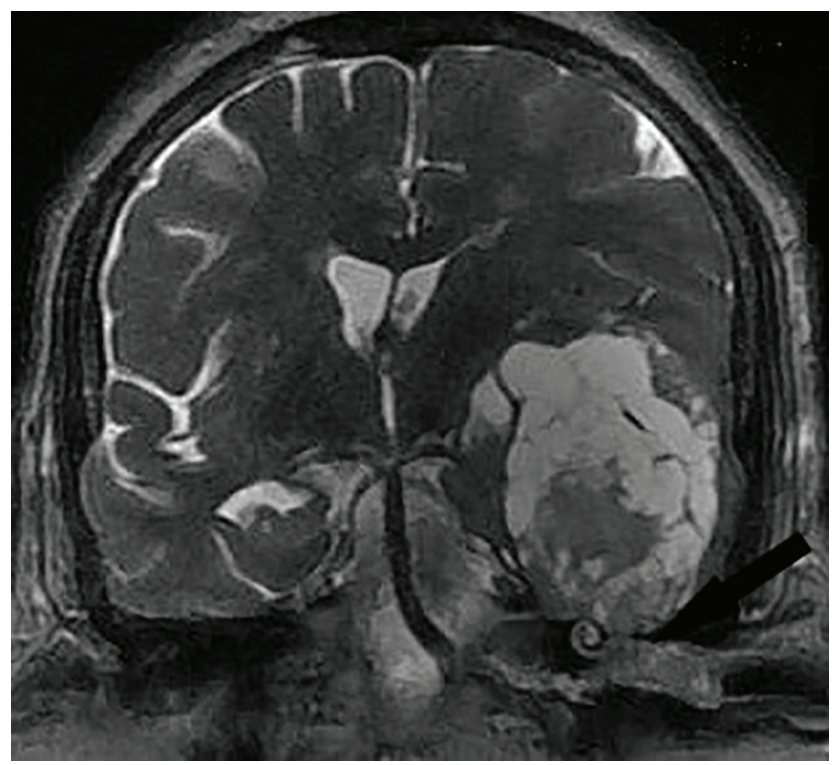

Fig. 3 Coronal T2W image showing a mixed solid-cystic lesion in the left temporal fossa. It perforates the tegmen tympani (arrow) and extends into the middle ear cavity and external auditory canal. Mass effect on the intracranial structures in the form of effacement of left lateral ventricle and mild contralateral midline shift is seen. T2W, T2-weighted 


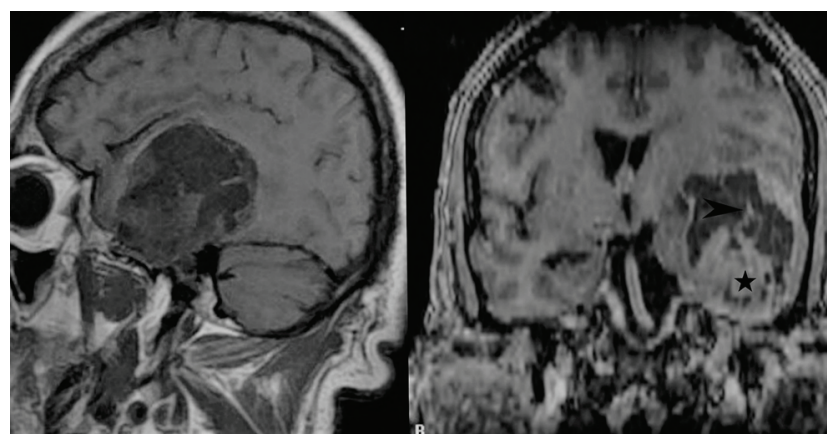

Fig. 4 (A) Sagittal pre-contrast T1W image showing temporal lobe $\mathrm{SOL}$ with isointense solid component and hypointense cystic component. (B) Coronal post gadolinium T1W fat saturated image showing homogenous enhancement of the solid component (asterix) and enhancing septae within the cystic component (arrow). SOL, space occupying lesion; T1W, T1-weighted.

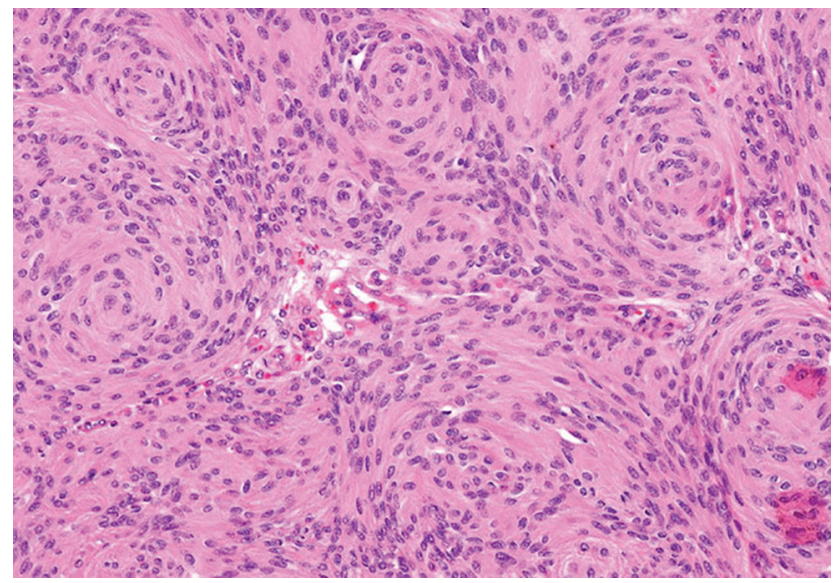

Fig. 5 Hematoxylin and eosin ( $\mathrm{H} \& \mathrm{E}$ ) sections showing meningothelial cells arranged in predominantly whorls and syncytial patternsuggestive of meningothelial meningioma.

\section{Discussion}

Meningiomas are nonglial neoplasms, which arise from arachnoid cap cells of the meninges. According to the WHO classification of CNS tumors published in 2016, ${ }^{2}$ there are 15 histological subtypes of meningioma. The most common variant is meningothelial meningiomas followed by fibrous and transitional meningiomas and together they account for approximately $80 \%$ of all meningiomas. Thus, these three subtypes could be regarded as typical meningiomas.

MRI is the modality of choice for diagnosis and characterization of meningiomas. The typical meningioma appears as a well circumscribed, homogeneous, vividly enhancing extra-axial mass. They characteristically appear hyperdense on non-contrast CT, iso- to hypointense on T1-weighted, and iso- to hyperintense on T2-weighted images. ${ }^{3}$ Most of the meningiomas do not show diffusion restriction or facilitation on diffusion-weighted images. ${ }^{4}$ Enhancing dural tail, hyperostosis of the underlying bones, linear internal flow voids, and calcification are also seen. ${ }^{5}$ Perilesional white matter edema is usually observed in large-sized tumors. MR spectroscopy (MRS) shows high alanine and low
$\mathrm{N}$-acetyl aspartate peaks in meningiomas; however, a major technical limitation of MRS in extra-axial tumors is their location near the skull and bone artifacts, thus making this technique limited mainly to large lesions. ${ }^{6}$ Meningiomas are highly vascular tumors, and in perfusion-weighted imaging, they show hyperperfusion with elevated rCBV values. ${ }^{7}$ On transcatheter angiograms, meningiomas show characteristic sunburst or spoke-wheel appearances. Also, the tumor contrast blush "comes early, stays late, and is very dense," popularly known as the mother-in-law sign. ${ }^{8}$

When these typical radiological features are present, the diagnosis of meningioma can be made with a high degree of certainty. However, sometimes even a histologically typical meningioma can show wide morphological diversity thus making the diagnosis confusing as in our case. The misleading imaging features encountered in our case were large cystic component with enhancing septae, significant perilesional edema, aggressive features such as bone erosion, and facial canal dehiscence. Due to the large size of the lesion and absence of characteristic extra-axial signs such as CSF cleft sign, broad dural base, dural tail, and displacement of blood vessels, it was not possible to decide on the intra-axial or extra-axial location of the tumor on routine sequences. The imaging features suggested an aggressive intracranial neoplasm. But, to our surprise, the histopathology came as WHO grade I meningioma.

On review of literature, we found a few case reports of temporal bone meningiomas extending to middle ear and causing otitis media. ${ }^{9,10}$ However, most of them had typical imaging features of en-plaque meningioma. Also, none of them showed aggressive behavior such as facial nerve palsy and bone erosion as in our case which makes it unique.

Complete surgical excision is the treatment of choice for meningiomas. In cases where complete resection is not possible, external-beam radiation therapy can be used. Tumor recurrence is a well-recognized complication of extracranial meningiomas. Vrionis et $\mathrm{a}^{11}$ reported a recurrence rate of approximately 54\% at 6 years of follow-up, despite gross total resection of temporal bone meningiomas. Therefore, patients treated for temporal bone meningiomas should be periodically followed up.

In conclusion, meningiomas can show atypical imaging features such as large meningeal cysts, heterogenous or ring enhancement, and aggressive features such as bone erosion. They can invade the middle ear and should be thought of while dealing with nonresponsive cases of otitis media. MRI brain with contrast should be done in nonresponsive cases of otitis media to rule out sinister intracranial etiology.

\section{Declaration of Patient Consent}

The authors certify that they have obtained all appropriate patient consent forms. In the form the patient(s) has/have given his/her/their consent for his/her/their images and other clinical information to be reported in the journal. The patients understand that their names and initials will not be published, and due efforts will be made to conceal their identity, but anonymity cannot be guaranteed. 


\section{Financial Support and Sponsorship}

None.

\section{Conflict of Interest}

None declared.

\section{References}

1 Whittle IR, Smith C, Navoo P, Collie D. Meningiomas. Lancet 2004;363(9420):1535-1543

2 Louis DN, Perry A, Reifenberger G, et al. The 2016 World Health Organization classification of tumors of the central nervous system: a summary. Acta Neuropathol 2016;131(6):803-820

3 Buetow MP, Buetow PC, Smirniotopoulos JG. Typical, atypical, and misleading features in meningioma. Radiographics 1991;11(6):1087-1106

4 Santelli L, Ramondo G, Della Puppa A, et al. Diffusion-weighted imaging does not predict histological grading in meningiomas. Acta Neurochir (Wien 2010;152(8):1315-1319, discussion 1319
5 Tokumaru A, O'uchi T, Eguchi T, et al. Prominent meningeal enhancement adjacent to meningioma on Gd-DTPA-enhanced MR images: histopathologic correlation. Radiology 1990;175(2):431-433

6 Cho YD, Choi GH, Lee SP, Kim JK, (. 1)H-MRS metabolic patterns for distinguishing between meningiomas and other brain tumors. Magn Reson Imaging 2003;21(6):663-672

7 Hakyemez B, Erdogan C, Bolca N, Yildirim N, Gokalp G, Parlak M. Evaluation of different cerebral mass lesions by perfusion-weighted MR imaging. J Magn Reson Imaging 2006;24(4):817-824

8 Osborn A, Tong K, Chapter IV, Intracranial neoplasms and tumor-like lesions. Handbook of Neuroradiology: Brain and Skull. 2nd ed. St. Luis: Mosby; 1996 220-250

9 Nicolay S, De Foer B, Bernaerts A, Van Dinther J, Parizel PM. A case of a temporal bone meningioma presenting as a serous otitis media. Acta Radiol Short Rep 2014;3(10):2047981614555048

10 Shihada R, Lurie M, Luntz M. Skull base meningiomas mimicking otitis media. J Laryngol Otol 2012;126(6):619-624

11 Vrionis FD, Robertson JH, Gardner G, Heilman CB. Temporal bone meningiomas. Skull Base Surg 1999;9(2):127-139 